\title{
EPISTEMOLOGÍA, ONTOLOGÍA Y DESAFÍOS DEL DERECHO
}

\section{EPISTEMOLOGY, ONTOLOGY AND CHALLENGES OF LAW}

Lucas Lavado Mallqui

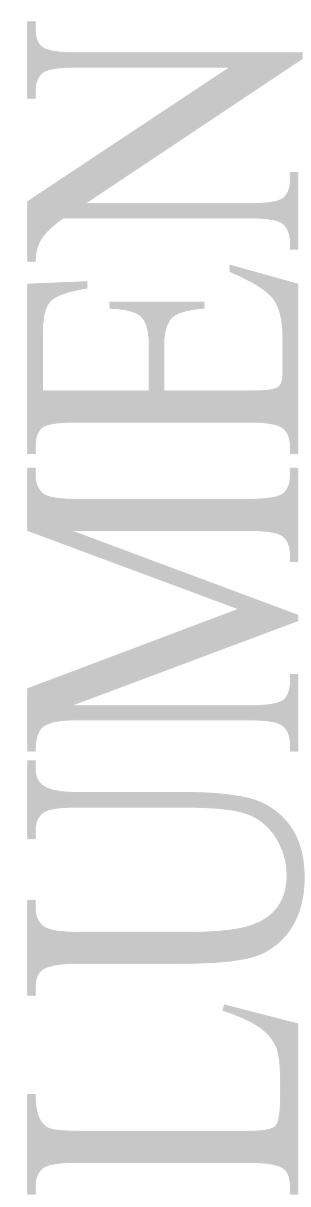




\title{
EPISTEMOLOGÍA, ONTOLOGÍA Y DESAFÍOS DEL DERECHO
}

\author{
EPISTEMOLOGY, ONTOLOGY AND CHALLENGES OF LAW
}

Lucas Lavado Mallqui

\begin{abstract}
Como se suele decir, ninguna norma se aplica de forma justa a hechos erróneos: como recordaba BENTHAM, la falsedad es la sirvienta de la injusticia.
\end{abstract}

Michele Taruffo (2010): Simplemente la verdad.

\section{RESUMEN:}

Este artículo abarca tres partes: la primera, algunas propuestas epistemológicas acerca de las teorías con énfasis en la teoría del derecho, teniendo en cuenta su estructura y los roles que cumple, principalmente respecto de la investigación. La segunda, incluye algunas precisiones acerca de la ontología de las normas jurídicas compatible con la epistemología. En tercer lugar, destaca los desafíos del derecho en el panorama actual enfatizando la interdisciplinariedad, la revisión de la metodología de la investigación y finalmente el rol de las facultades de derecho respecto de la eficiencia de la justicia en el Perú.

\section{PALABRAS CLAVE:}

Epistemología, conceptos, teoría, ontología, metodología, interdisciplina.

\begin{abstract}
:
This article covers three parts: the first, some epistemological proposals about the theories, with emphasis on the theory of law taking into account its structure and the roles it fulfills mainly with respect to research. The second, includes some details about the ontology of legal norms compatible with epistemology. Third, it highlights the challenges of law in the current scenario, emphasizing interdisciplinarity, the review of research methodology and finally the role of law schools regarding the efficiency of justice in Peru.
\end{abstract}

\section{KEY WORDS:}

Epistemology, concepts, theory, ontology, methodology, interdiscipline.

\section{Introducción:}

\section{Epistemología de las teorías}

La epistemología o gnoseología es una disciplina filosófica que, entre otros problemas relevantes se interesa por el análisis de los conceptos científicos, los problemas, las teorías y la verdad, entre las cuestiones de actualidad. Su importancia es de primer orden para abordar y analizar las diferentes perspectivas que subyacen no solo a los currículos formación de abogados sino de las grandes decisiones que atañen a los que tienen en sus manos la administración de justicia. Por ejemplo, los debates entre los que se ciñen a la "íntima convicción" y las pautas objetivas y la pruebas, entre los prácticos y los teóricos, en fin, entre los que defienden la argumentación sustentada en la lógica y los que se preocupan principalmente por la persuasión.

Comenzar por la aclaración del concepto de teoría puede ayudar. Hay consenso acerca de que las teorías científicas son sistemas de ideas organizadas lógicamente y guiadas por la coherencia 
y el realismo. Permiten, por lo tanto, explicar y comprender el mundo en que vivimos, asumir un enfoque y tomar decisiones enmarcadas en una visión.

Constituyen los aportes más sobresalientes de las ciencias. Aquellos cerebros que han inventado y propuesto teorías han dado pasos innovadores para el incremento del desarrollo científico y sentado las bases para posteriores descubrimientos en diferentes campos del saber.

Sin embargo, ocurre con frecuencia el error de confundirla con la charlatanería y la verborrea, con la palabrería o divagación sobre cualquier tema. Esto ha conducido a la desconfianza y el menosprecio de las teorías, incluso en ámbitos más o menos cultos que pregonan el valor de la práctica por sobre la teoría, debido al desconocimiento de que las prácticas y las tecnologías se fundan en ellas. Las teorías son arquitecturas conceptuales rigurosas, por lo que antes de contestar en qué consisten y para qué sirven, convendría registrar algunos datos y un esquema de su composición o estructura.

La sola mención del término teoría, de inmediato nos pone en presencia de conquistas científicas gigantes que han transformado nuestra manera de entender y transformar el mundo y a nosotros mismos: Teoría del Big Bang, Teoría de la Relatividad, Teoría de la Evolución, etc. Existen también teorías en campos más restringidos como el derecho: Teoría Pura del Derecho de Hans Kelsen, Teoría del proceso de Michele Taruffo, Teoría de la autoría mediata del delito de Claus Roxin, Teoría garantista de Luigi Ferrajoli, etc. Una manera de estimar su importancia es constatar su impacto en la sociedad, las citas y referencias que se hacen acerca de sus obras y una comunidad científica trabajando en institutos y universidades siguiendo su impronta o replanteándolas.

La respuesta acerca de la pregunta sobre sus roles, constituye una larga lista que no es el momento de hacerla, bastaría poner el acento en algunos. En primer lugar, gracias a las teorías podemos ver cosas, hechos y procesos de nuestro entorno y dentro de nosotros mismos, que sin ellas estaríamos ciegos, para decirlo figuradamente, e imposibilitados para explicarlos y entenderlos. En segundo lugar, orienta el conocimiento y la investigación científica gracias a su andamiaje conceptual. En tercer lugar, permite analizar problemas complejos y dirigir la práctica. Su fortaleza radica en la manera de cómo está construida, es decir, en su estructura a partir de unidades pequeñas de ideas hasta lograr una arquitectura mayor. La simplicidad del esquema, puede ayudar a analizar el conjunto constitutivo de sus elementos.

Esquema 1. Concepto-proposición.teoría

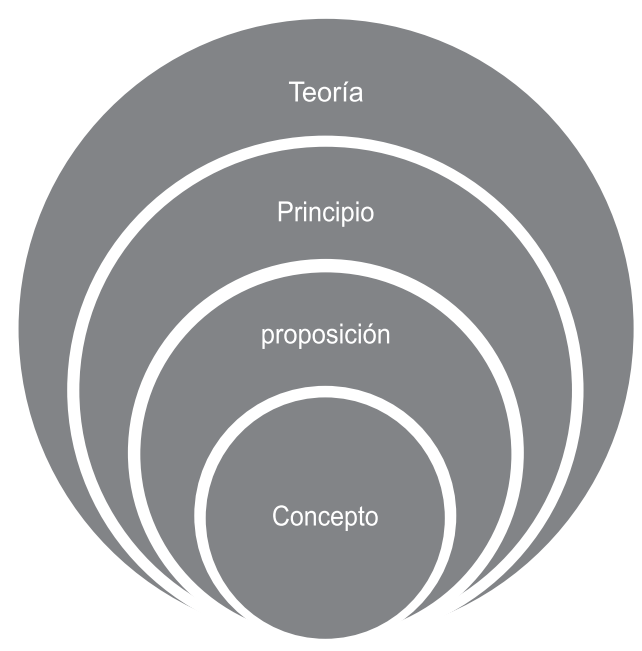


Las teorías son sistemas de proposiciones articuladas en base a la lógica, cuidando su coherencia y su correspondencia con los hechos. Son expresiones cuyas características esenciales son las de ser verdaderas o falsas y se construyen sobre la base de los conceptos (sus unidades constitutivas más pequeñas). Los conceptos son unidades de significación con los cuales se piensan las cosas sobre las que se teorizan. Ejemplo los conceptos de norma, jurisprudencia, justicia, prueba, etc. El esquema -visto de manera descendente- muestra que las teorías incluyen proposiciones (los principios e hipótesis tipos de proposiciones) y conceptos.

Para ganar en precisión debemos valernos de la epistemología más exitosa y para expresarlo de manera técnica adoptaremos la propuesta del profesor Mario Bunge (1999) para quien teoría es un "sistema hipotético-deductivo, es decir un sistema de proposiciones algunas de las cuales tienen forma de hipótesis y el resto son deducciones a partir de premisas [...] Las teorías no son hipótesis sino sistemas de hipótesis" (p.165). Si se mira el gráfico - de manera ascendente- se verá que la unidad mínima de construcción de una teoría es el concepto, luego vienen las proposiciones -hay que tener en cuenta que las hipótesis son proposiciones- finalmente el constructo mayor que es la teoría.

\section{La teoría del derecho}

No existe, en la actualidad, unanimidad sobre las definiciones de teoría del derecho y de la norma jurídica, sin embargo, la lectura de algunas contribuciones relevantes, permite sostener que avanza hacia una convergencia inevitable.

Hans Kelsen (2011) en 1934 delimitó el campo y estableció sus límites al "definir el derecho como norma y restringir la ciencia jurídica [...] al conocimiento normativo" (p. 46). En 1960 imparte cuatro conferencias en la Universidad Nacional Autónoma de México (2009), para presentar su teoría que constituye un hito. A sus 79 años, con el rigor intelectual que le caracterizaba, inicia su exposición como un desafío que aún resuena en las inteligencias más lúcidas: "La Teoría Pura del Derecho, como su nombre lo indica, es una teoría del Derecho. La forma por la que se elabora una teoría está determinada por su objeto" (p. 15). Esta sola afirmación es más que suficiente para un seminario especialmente diseñado. Renglón seguido, después de descartar el enfoque del derecho cuyo objeto son los hechos, se pronuncia a favor del derecho como norma que tiene el carácter de orden, pero también de autorización y de permiso. Lo que ha venido después ha sido el análisis, el debate y el avance a partir de este hito.

Desde sus famosas exposiciones en la UNAM han transcurrido 59 años. La bibliografía en ciencia jurídica y filosofía del derecho se ha multiplicado exponencialmente, lo que permite encontrar derroteros. Desde la perspectiva del profesor Luigi Ferrajoli (2008) por teoría del derecho se entiende "sumariamente, el conjunto de cosas y/o de experiencias de las cuales la (o una determinada) teoría del derecho habla [...], como una teoría cuyos términos y cuyas tesis mantienen una relación semántica con un determinado ámbito de la experiencia o de la realidad" (p. 17). Una teoría que incluye un aspecto de la realidad históricamente constatable tanto en su campo como su contenido al que se le llama "derecho". En este sentido puede hablarse de una teoría en general, así como de la teoría del Derecho de un país. El profesor Ferrajoli (2017), sin duda, sabe que las teorías en todos los campos científicos ocupan un estatus especial debido a la potencia explicativa que radica en su coherencia y, es lo que le ha llevado en uno de sus trabajos recientes a elucidar diez aporías en la Teoría Pura del Derecho de Hans Kelsen, con los que le da "un vuelco a la tesis a la tesis kelseniana de la no aplicabilidad de la lógica al derecho" (p.12). De otro lado, el profesor Joseph Raz (2007) muestra otros aspectos no menos importantes de las teorías, tales que revisten especial interés para orientar investigaciones en lo que concierne al marco teórico e hipótesis, de las que carecen los manuales que circulan en el medio y que resultan algo extraños al desarrollo real de esta disciplina y sus cultores. Para el profesor Raz la teoría del derecho tiene como tarea: 
"[Proporcionar] una explicación de la naturaleza del derecho. La tesis que defenderé sostiene que una teoría del derecho es exitosa si satisface dos criterios: en primer lugar, si consiste en proposiciones acerca del derecho que son necesariamente verdaderas $\mathrm{y}$, en segundo lugar, si estas proposiciones explican lo que es el derecho" (pp. 47-48).

Es bueno detenerse un tanto en el énfasis que pone el autor sobre las proposiciones y los conceptos que constituyen hoy aspectos centrales del análisis en las investigaciones filosóficas del derecho.

\section{Conceptos}

Las reflexiones anteriores nos llevan a la necesaria aclaración de lo que son los conceptos, por tres razones principales: a) el análisis conceptual ocupa un lugar destacado en los estudios jurídicos actuales, b) ayuda a la argumentación y la búsqueda de fundamentación racional en el debate y, c) contribuyen con la adecuada articulación de las ideas y la construcción de una cosmovisión racional.

Las ciencias sociales, a diferencia de las naturales como la física y la química, le destinan atención especial a la aclaración de conceptos en busca de precisión y rigor. Los conceptos permiten construir proposiciones y éstas organizan con ellas una trama articulada que es la teoría, como se ha dicho antes. Quien quiera pensar e investigar en filosofía del derecho está constreñido a refinar y aclarar sus conceptos como por ejemplo norma, hecho, verdad, prueba, acceso a la justicia, argumentación y otros que hacen posible su desarrollo como disciplina. El debate sigue su curso de modo que un filósofo del derecho como el profesor Robert Alexy (2016) retoma y aclara su tesis de la "doble naturaleza del derecho" para enfatizar que la "filosofía es razonamiento acerca del razonamiento". El profesor Ernesto Garzón Valdés (2014) desde una postura analítica y congruente con su lucha contra el oscurecimiento conceptual tiene un mensaje que bien vale la pena procesar:

[Pienso que no es muy aventurado sugerir que la tarea que un filósofo del derecho latinoamericano tiene que asumir es doblemente complicada: por una parte, no puede dejar de tomar en cuenta, entre otros, los problemas universales de esta disciplina; por otra, tiene que tratar de desgarrar el velo de la retórica y esquivar la trampa de la ciega imitación de modas filosóficas [...] No hay, pues, que propiciar el rechazo cerril del pensamiento ajeno ni estimular su frívola recepción acrítica (p.17).

El análisis ocupa un lugar central y el análisis de los conceptos es el inicio porque son unidades de significado con los que se comienza. Como se ha dicho antes, sin conceptos no se pueden construir proposiciones y sin éstas no se pueden formular teorías. No está demás añadir que las proposiciones o enunciados son expresiones sujetas a los criterios de verdadero-falso. El concepto de justicia ha ocupado lugar prominente del pensamiento filosófico del profesor John Rawls (1997) que le ha dedicado sus mejores esfuerzos en la formulación de su teoría tan difundida e influyente en el siglo XX. Sin embargo, tal empresa no quedó allí, como docente universitario autocrítico, lo fue repensando a lo largo de sus clases, para mostrar finalmente una reformulación de su obra original (2002). De donde se desprende que la filosofía es pertinente al derecho, es más, los conceptos jurídicos más importantes también son filosóficos. Norberto Bobbio (Fernández Santillana, 1996) propone un análisis muy sugestivo de los pares de conceptos siguientes: justicia-ley, justiciaigualdad y justicia-orden (p. 204).

Uno de los mejores aportes del filósofo Jesús Mosterín (2000) está referido a la distinción entre el aparato perceptual y conceptual y su diáfana aclaración sobre los conceptos con los que pensamos, inevitablemente atadas a las palabras con las que hablamos, sin descuidar por cierto relacionarlas. Gracias al uso adecuado de los conceptos es posible pensar con claridad y a la elección pertinente 
de las palabras podemos expresarnos con precisión. La metáfora del profesor Joseph Raz (2007) es aquí útil cuando dice "[hablando metafóricamente, los conceptos [...] están situados entre el mundo, de cuyos aspectos son conceptos, y las palabras o frases que los expresan (a los conceptos) y son empleadas para hablar sobre esos aspectos del mundo" (p. 49). Otra vez, un esquema puede ayudar.

\section{Esquema 2. Palabras-concepto-mundo}

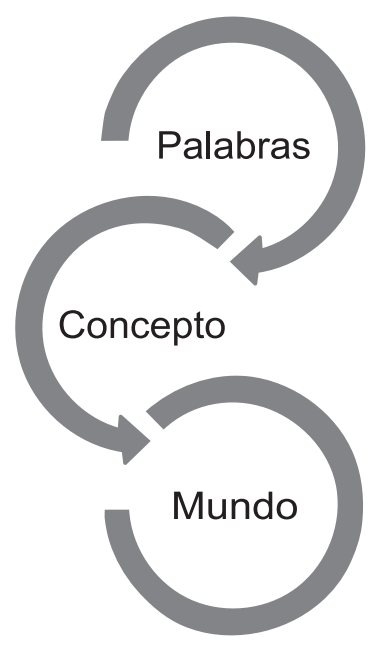

Entonces, el análisis conceptual en derecho o en cualquier otra disciplina, no es comparable al análisis terminológico o aclaración del significado de las palabras o frases. Para esto vale el Diccionario de la Real Academia. Más concisamente "Los conceptos consisten en cómo concebimos aspectos del mundo, y se encuentran entre las palabras en las cuales son expresados y sus significados, por un lado, y la naturaleza de las cosas a las que se aplican, por el otro" (Raz, cit., p. 50). Pese a la sistemática resistencia de ciertos círculos académicos, esta misión es impostergable en el diseño de las investigaciones sobre todo cuando se organiza el marco teórico y conceptual y, sobre todo, cuando se formulan y se operacionalizan hipótesis. En fin, el que ayuden a pensar ya es de alguna importancia.

El buscar y aclarar conceptos es una aventura intelectual placentera. Acrecienta nuestra manera de entender el mundo, están en las palabras de las que nos valemos para expresarlos, hacen referencia a las cosas a las que se aplican. A partir de aquí, lo entenderemos si entendemos los rasgos de las cosas a las que se refieren. En consecuencia, a nadie escapará que el concepto de norma (entre tantos otros, a decir verdad, no muchos) es fundamental en derecho y seguramente una vida entera no será suficiente solo para leer lo que se ha pensado y escrito sobre ella.

\section{Ontología}

En primer lugar, la ontología es una disciplina filosófica cuyo propósito es el estudio de la realidad y su estructura cambiante, el tiempo, las relaciones de causa a efecto. Para este caso asumiremos supuestos ontológicos tan generales como que los hechos sociales son realidades existentes independientes de nuestra voluntad, que trascurren en el tiempo y en consecuencia están sometidos a cambios tanto cuantitativos como cualitativos. Los supuestos ontológicos subyacen en la investigación científica y es necesario partir de ellos para no perderse en especulaciones y trivialidades. Le atañen también otros problemas de interés como son las diferencias entre las 
leyes científicas y las reglas sociales, los procesos de cambio social y la ruptura de las normas legales. Despejar malos entendidos como el de entender que el derecho no crea hechos, sino que los regula, lo que se olvidan con frecuencia sobre todo cuando se investiga y se legisla.

Siguiendo al profesor Bunge (1989), una sociedad es en primer lugar un sistema concreto compuesto por individuos conectados entre sí, en segundo lugar, toda sociedad tiene propiedades sistémicas o emergentes como los regímenes políticos, que sobrepasan las propiedades individuales y, en tercer lugar, la sociedad no puede actuar sobre sus miembros, así como el organismo no puede actuar sobre sus células (p. 133). Estos principios ontológicos están en la base de los sistemas jurídicos y como sostiene Manuel Atienza (2017) puede contribuir a la comprensión del Derecho como una herramienta de transformación, porque al derecho le concierne "la organización de la sociedad. Y que los saberes jurídicos (incluida la filosofía del Derecho) bien pueden considerarse también como saberes de tipo organizativo" (p. 361).

Es importante prestar atención a las relaciones y, por cierto, diferencias entre teoría y realidad y consecuentemente a la ontología de las normas que podría ser de ayuda para algunas reflexiones. Son cuestiones abiertas al diálogo y al debate algunas interrogantes ontológicas sobre las normas: ¿las normas jurídicas existen y, si existen, en qué sentido existen? Las primeras respuestas subyacen a las teorías de Kelsen, Ferrajoli, Raz y H. L. A. Hart (1998).

Las preguntas por la ontología del derecho o cualquier campo de las disciplinas sociales, no son simples, aunque a primera vista puedan parecer tales, puesto que no puede decirse que las normas existen en el mismo sentido en que existen los automóviles, los puentes o las casetas de peaje e incluso las organizaciones políticas. Lo cierto es que existen y es necesario aclarar su estatus ontológico. Ocurren confusiones derivadas de otros errores categoriales muy difundidos y la falta de persistencia en aclararlas. Es posible que estos errores categoriales se gestan cuando autores de enjundiosos "tratados" utilizan las definiciones verbales en lugar de análisis conceptuales.

Vivimos en un mundo de normas cuyas diferencias saltan a la vista con solo mencionarlas como es el caso de los siguientes cuatro tipos: normas morales, normas religiosas, normas técnicas y normas jurídicas. En primer lugar, se admite que las normas son sistemas conceptuales, en segundo término, para responder en qué sentido se predica de su existencia, se asume aquí la propuesta del profesor uruguayo Daniel Mendonca (2008, pp. 48-52) cuyo enfoque es ampliamente conocido.

1. Las normas existen cuando son acatadas, de modo que Kelsen (2011) lo definiría como eficacia, es decir cuando se cumplen (p. 23), que además es condición de su validez.

2. Las normas existen cuando pertenece a un determinado sistema de normas (ordenamiento). Una norma aislada y fuera del sistema no sería tal.

3. Las normas existen si es que son obligatorias. Cuando una autoridad superior la emite disponiendo su obligatoriedad de cumplimiento.

4. Las normas existen cuando han sido formalmente promulgadas por una autoridad competente.

5. Las normas existen cuando han sido formuladas lingüísticamente. Tiene un contenido conceptual que es independiente del lenguaje.

Es, sin duda, el inicio, hace falta abrir líneas de investigación. Un debate sobre la ontología ayudaría a enriquecer la epistemología del derecho, sobre todo en la reconstrucción de la epistemología normativa o metodología de la investigación que comienza a discutirse desde diferentes enfoques (Lariguet 2016). Las normas no están solas sino formando relaciones denominadas ordenamiento. En el marco de la teoría del Derecho, la teoría de las normas es una parte integrante de aquél. 


\section{Desafíos}

Visto desde la perspectiva de la epistemología y la ontología, el Derecho forma parte del sistema científico y tecnológico. No es posible concebirlo como una disciplina inconexa y aislada. De este modo el mundo de la ciencia y de la tecnología, donde está inmerso el derecho, influido en mayor o menor medida por las innovaciones veloces presionan no solamente sobre la definición de las competencias profesionales sino de las instituciones, produciendo cambios sistémicos.

Entonces, no es difícil entender que la profesión de abogado se desenvuelve en un mundo de mutaciones sociales y tecnológicas de enormes proporciones como la informática, la robótica, las biotecnologías e Internet. Ya pueden percibirse los efectos que está produciendo en el derecho las tecnologías sociales y naturales: no solo en el surgimiento de nuevas especialidades y su impacto en el campo de la administración de justicia, sino en las innovaciones de impacto tecnológio. Es, por ejemplo, alarmante lo que el profesor de Harvard Yochai Benkler (2019) señala en el sentido de que la industria puntera de Norteamérica está influyendo para dictar "la ciencia, la moral y las leyes de la inteligencia artificial" (p.161) a fin de decidir el futuro de las investigaciones tecnológicas limitando la libertad de investigación en beneficio del empoderamiento de las grandes empresas con creciente poder.

Estamos frente a una gama de desafíos para el abogado del siglo XXI, que son abordados por científicos del derecho y filósofos, quienes con sus investigaciones contribuyen a ensanchar su campo e influencia en la sociedad. Seguramente no estaba en la agenda de aquel portento intelectual que fue Becaria, cuando en 1764 escribió su De los delitos y las penas. Ni de Hans Kelsen, cuando en 1934 publica su Teoría Pura del Derecho. Son otras épocas.

Las preguntas no se hacen esperar: ¿son lo mismo ciencia y tecnología?, ¿guardan alguna relación? La respuesta es que no son lo mismo, aunque se relacionan. La ciencia explica lo que ocurre en el mundo, en cambio la tecnología se preocupa por su transformación, control y regulación. Un ejemplo trivial es, por ejemplo, la sucesión de los días y las noches, es decir, por qué se producen. En cambio, la tecnología se plantea la cuestión de cómo iluminar las viviendas, las fábricas y las ciudades en áreas rurales a costos razonables. La primera es científica y la segunda práctica. La primera reclama conocimiento y la segunda acción (Lavado, 2016)

Se suele confundir tecnología con máquinas y artefactos. No solo es un error importante sino peligroso, porque lleva a pensar que, si se compra o se alquila, como país, artefactos y maquinarias sofisticadas en los campos educativo y militar, por ejemplo, se estaría pensando equivocadamente en un progreso en tecnología educativa y militar. No es así, los artefactos son objetos resultantes del diseño de prototipos, pruebas exigentes, fabricación y finalmente las patentes y la comercialización. La tecnología consiste en este proceso complejo cuyo centro es el diseño. Vivimos hoy en la era del diseño. Los artefactos tecnológicos pronto serán obsoletos, muchas veces antes de que sepamos siquiera repararlos, adaptarlos o modificarlos, porque la posesión de la tecnología no solo consiste en la posesión de conocimientos tecnológicos y de centros de investigación que los conciba, diseñe y desarrolle, sino de la defensa de las patentes para su fabricación (Lavado, 2018).

El derecho está impelido a ampliar su horizonte de conocimientos y de acción: la informática, las prótesis cognitivas, la identidad digital, la bioética, el aborto terapéutico, la filiación, la concepción asistida, la biotecnología, los datos masivos o big data, etc. Se comienza a enfrentar los desafíos de la medicina genómica de la que da cuenta Jennifer Couzin-Frankel (2019) que "los médicos y otros proveedores de atención médica ya están enfrentando demandas legales que abordan un nuevo terreno Legal y, a veces, incluso los hacen responsables por la forma en que ofrecen, interpretan y asesoran a los pacientes sobre las pruebas genéticas" (p. 521). 
También se producen movimientos tradicionalistas: la homeopatía ubicada en el "limbo legal", acaba de tocar a su fin, dado que ya se le aplica la directiva europea de 2001 "que regulariza definitivamente la situación de los remedios homeopáticos en el mercado de medicamentos" (El País, 11.5.14, p. 36). Ocurre, pese a que es perjudicial para los pacientes oncológicos tras optar por este tratamiento denunciado por el biólogo Vicente Prieto (2018) para quien los "remedios homeopáticos son delirantes. El más común es tratar la depresión con muro de Berlín diluido. Según ellos te tienes que tomar algo que te produzca los mismos efectos" ( $p$. 48). Es lo que hoy se denomina pseudo ciencias y pseudo tecnologías que socavan la verdad y la eficiencia. Un campo multidisciplinar que no escapa al derecho. En este contexto no es difícil vislumbrar algunos desafíos importantes que merecen ser considerados como parte de las líneas de investigación de los institutos de investigación.

Primero: Vencer el cerco disciplinar, una muralla edificada para impedir que las especialidades jurídicas vivan y convivan aisladas de las demás ciencias a espaldas de un principio básico: "dada una disciplina científica existe una interdisciplina que la vincula a otra disciplina científica" (Bunge, 2001). La biología molecular, la informática, la robótica, la física, la nanotecnología, la cosmología, etc., están obligando al derecho a plantear nuevas especialidades, que de no hacerlas estarían dejando fuera de sus dominios nuevas realidades y nuevos problemas. En este sentido tocan las puestas del derecho el cambio climático, la medicina genómica, la inteligencia artificial, la diversidad biológica. La crítica académica seria sobre el aislamiento disciplinar persiste (Capelletti y Garth 1996, Taruffo 2006, Ferrajoli 2016).

Segundo: Revisión y rediseño del enfoque metodológico de las asignaturas de investigación de los currículos de derecho y de las líneas de investigación de los institutos para proponer nuevas alternativas. Es un hecho verificable que los manuales de investigación en derecho que circulan proficuamente están alejados de las investigaciones y del aporte de que se producen en las disciplinas mismas. Una mirada a los conceptos centrales tales como el planteamiento de problemas, hipótesis, marco teórico y métodos de contrastación de hipótesis, permite constatar una evidente enajenación (Ramos Núñez 2014, Palacios Vilela, Romero Delgado y Ñaupas Paitán, 2016) respecto de los debates y contribuciones actuales

Tercero: Superar de manera drástica la disonancia entre los problemas de corrupción que afecta a la sociedad peruana y la respuesta de las facultades de derecho utilizando sus posibilidades académicas y recursos disponibles. Un antecedente de impacto, que con el tiempo ha ido adquiriendo mayor peso se produjo hace aproximadamente 15 años cuando el investigador Luis Pásara (2004) publicó un informe encargado por el Ministro de Justicia de entonces. El problema central consistió de averiguar el impacto de la enseñanza del derecho en el Perú sobre la administración de justicia. Una de sus conclusiones es elocuente:

La interrogante central es si existe en las facultades de derecho, y en las universidades en general, disposición a emprender el camino del cambio y evaluar la utilidad de sus programas de estudios. Si se mira a lo ocurrido en las dos últimas décadas, todo parece indicar que una respuesta afirmativa resulte poco realista (p.93).

Aquella investigación sigue siendo un modelo para encaminar la investigación universitaria y fue posteriormente incluida por el autor (2010) conjuntamente con otros estudios coadyuvantes. Sin embargo, este tercer desafío, pese a los peligros de la política doméstica, se está encarando en el marco de la nueva Ley Universitaria vía el licenciamiento y la acreditación. Luego, conviene advertir que no está exenta del peligro de distorsión y desaparición a causa de factores externos. 


\section{Resumen}

La epistemología es una disciplina filosófica que estudia los fundamentos de las ciencias, le interesa los problemas científicos, las teorías, el sistema científico y tecnológico y las relaciones interdisciplinares entre otras cuestiones relevantes. En este sentido las teorías con constructos que permiten explicar la realidad y están constituidas por conceptos y proposiciones. Los conceptos son unidades de significado que permiten formular proposiciones y constituyen objetos privilegiados del análisis. La ontología es la teoría de la realidad y es congruente con la epistemología, en este sentido la ontología de las normas permite elucidar en qué sentido se entiende la existencia de las normas y cuál es su lugar. Finalmente, en este contexto, algunos desafíos del derecho son la interdisciplinariedad, la reorientación de los métodos de investigación, la congruencia entre la formación universitaria de las competencias profesionales del abogado y la eficiencia de la justicia peruana.

\section{REFERENCIAS}

- $\quad$ Alexy, R. (2016). La doble naturaleza del derecho. Madrid: Trotta.

- Atienza, M. (2017). Filosofía del derecho y transformación social. Madrid: Trotta.

- $\quad$ Benkler, Y. (2019). Don't let industry write the rules for All. Nature Vol 569, Issue No 7755.

- $\quad$ Bunge, M. (1989). Mente y sociedad. Madrid: Alianza Editorial.

- Bunge, M. (1999). Buscar la filosofía en las ciencias sociales. Buenos Aires: Sudamericana.

- Bunge, M. (2001). Diccionario de filosofía. México, D.F: Siglo Veintiuno Editores.

- Cappelletti, M. y Garth, B. (1996). El acceso a la justicia. La tendencia en el movimiento mundial para hacer efectivos los derechos. México, D.F.: Fondo de Cultura Económica.

- Fernández Santillana, J. (1996). Norberto Bobbio: el filósofo y la política (antología). México, D. F: Fondo de Cultura Económica.

- Ferrajoli, L. (2008) Epistemología jurídica y garantismo. México, D. F.: Distribuciones Fontamara, S.A.

- Ferrajoli, L. (2016). Los derechos y sus garantías: conversación con Mauro Barberis. Madrid: Trotta.

- Ferrajoli, L. (2017). La lógica del derecho: Diez aporías en la obra de Hans Kelsen. Madrid: Trotta.

- Garzón Valdés, E. (2011). Propuestas. Madrid: Trotta.

- Hart, H. L. A. (2012). El concepto de derecho. Buenos Aires: Abeledo-Perrot.

- Kelsen, H. (2009) [1960]. Introducción a la Teoría Pura de Derecho. México, D.F: Ediciones Coyoacán.

- Kelsen, H. (2011) [1934]. Teoría Pura del Derecho. Introducción a los problemas de la ciencia jurídica. Madrid: Trotta.

- Kouzin-Frankel, J. (2019). Genomics breeds new legal questions. Science, Vol 364, Issue 6440, p. 521

- Kouzin-Frankel, J. (2019). Genomics breeds new legal questions. Science, Vol 364, Issue 6440 , p. 52

- Lariguet, G. (2016). Metodología de la investigación jurídica: propuestas contemporáneas. Córdova (Argentina): Brujas. N 30220

- Lavado, L. (2016). Epistemología e investigación: a propósito de la Ley Universitaria $\mathrm{N}^{\circ}$ 30220. Vox Juris, Vol. 32, N²,106-115.

- Lavado, L. (2018). Métodos en ciencias sociales: cinco propuestas. Lima: Grijley.

- Mendonca, D. (2008). Las claves del derecho. Barcelona: Gedisa.

- Mosterín, J. (2000). Conceptos y teorías en la ciencia. Madrid: Alianza.

- Palacios Vilela, J.J., Romero Delgado, H.E. y Ñaupas Paitán, H. (2016). Metodología de la 
investigación jurídica: una brújula para investigar en ciencias jurídicas y redactar tesis. Lima: Grijley.

- Pásara, L. (2004). La enseñanza del derecho en el Perú: su impacto sobre la administración de justicia. Lima: Ministerio de Justicia.

- Pásara, L. (2010). Tres claves de la justicia en el Perú: Jueces, justicia y poder en el Perú, La enseñanza del derecho, Los abogados en la administración de justicia. Lima: Fondo Editorial de la PUCP

- $\quad$ Prieto, V. (enero 16, 2018). Me he suicidado dos veces con homeopatía. El Mundo, p. 48

- Ramos Núñez, C. (2014). Cómo hacer una tesis de derecho y no envejecer en el intento. Lima: Grijley

- Rawls, J. (1997). Teoría de la justicia. México, D.F.: Fondo de Cultura Económica.

- Rawls, J. (2002). La justicia como equidad: una reformulación. Barcelona: Paidós de Espasa Libros.

- Raz, J., Alexy, R. y Bulygin, E. (2007). Una discusión sobre la teoría del Derecho. Madrid: Marcial Pons Ediciones Jurídicas y Sociales.

- Taruffo, M. (2006). Sobre las fronteras: escritos sobre la Justicia Civil. Bogotá: Norma.

Fecha de recepción: 30 de abril de 2019

Fecha de aceptación: 16 de junio de 2019 